\title{
The Overlapping Running Suture Method Using Single Knotless Barbed Absorbable Suture Material for Abdominal Wall Closure after Single Incision Laparoscopic Appendectomy: Comparison with the Traditional Interrupted Closure Technique
}

\author{
Dong Hyun Kim, M.D., Jung Ho Park, M.D., Jung II Joo, M.D., Jang Yong Jeon, M.D., Ph.D., Sang Woo Lim, M.D., Ph.D. \\ Department of Surgery, Hallym University College of Medicine, Hallym University Sacred Heart Hospital, Anyang, Korea
}

Purpose: The aim of our study was to present an abdominal wall closure technique using barbed suture $\mathrm{V}-\mathrm{Loc}^{\mathrm{TM}} 90$ after single incision laparoscopic appendectomy (SILA) and to compare perioperative outcomes with conventional layer by layer abdominal wall closure after SILA.

Methods: From March 2014 to July 2016, a retrospective case-control study was conducted for a total of 269 consecutive patients who underwent SILA. According to abdominal wall closure methods, 129 patients were classified into the V-Loc closure group and 140 patients were assigned into the conventional layer by layer closure group. In the V-Loc group, abdominal wall closure was performed from the fascia to the skin with a single thread of unidirectional absorbable barbed suture V-Loc ${ }^{\mathrm{TM}} 90$ 2-0 using continuous running suture and reverse overlapping reinforced running technique. Subcutaneous closure and subcuticular suture were performed with the remaining portion of V-Loc.

Results: The V-Loc closure group showed shorter total operation time (40.0 \pm 15.4 min vs. $44.9 \pm 16.3$ min, $p=0.013$ ) and abdominal wall closure time ( $5.5 \pm 0.9 \mathrm{~min}$ vs. $6.5 \pm 0.8 \mathrm{~min}, p<0.001$ ). Postoperative incision length was significantly shorter in the V-Loc closure group $(1.1 \pm 0.3 \mathrm{~cm}$ vs. $1.8 \pm 0.4 \mathrm{~cm}$, $p<0.001$ ). Postoperative wound pain, time to resume diet, postoperative hospital stay, complications including surgical site infection, or mean patient satisfaction score at one month after hospital discharge was not significantly different between the two groups.

Conclusion: In conclusion, unidirectional knotless barbed suture is a safe alternative method for abdominal wall closure after SILA. It can save time while providing comparable cosmesis.

Keywords: Laparoscopy, Appendectomy, Suture technique
Received June 27, 2018

Revised 1st August 11, 2018

2nd September 3, 2018

Accepted September 4, 2018

Corresponding author

Sang Woo Lim

Department of Surgery, Hallym University College of Medicine, Hallym University Sacred Heart Hospital, 22 Gwanpyeong-ro 170gil, Dongan-gu, Anyang 14068,

Korea

Tel: +82-31-380-3772

Fax: +82-31-380-1619

E-mail: swlim12@hallym.ac.kr ORCID:

http://orcid.org/0000-0001-8248-8406

\section{INTRODUCTION}

Laparoscopic approach in acute appendicitis has several advantages over the open procedure, including faster recovery, better patient satisfaction, less wound related complications, and less postoperative adhesions. ${ }^{1}$ In addition, single incision laparoscopic surgery can be applied. Single incision laparoscopic appendectomy (SILA) has been widely performed with acceptable outcomes, including similar postoperative recovery compared to conventional three-port laparoscopic appendectomy. $^{2}$

There has been improvement in the method of wound clo- 
sure after appendectomy to reduce wound-related complications including surgical site infections and trocar site hernia while providing better cosmetic results. ${ }^{3-6}$

Recently, knotless barbed absorbable sutures have been used in various surgical situations, including gastrointestinal surgery, ${ }^{7-10}$ gynecologic surgery, ${ }^{11}$ and hepatobiliary-pancreatic surgery. ${ }^{14-16}$

Currently, V-Loc (Covidien, Mansfield, MA, USA), an absorbable wound closure device that is US Food and Drug Administration (FDA)-approved barbed suture, is a unidirectional barbed suture with a needle at one end and a welded loop at the other end. ${ }^{17}$ We applied V-Loc ${ }^{\mathrm{TM}} 90$ 2-0 for abdominal wall closure after SILA. The aim of our study was to present our experience of abdominal wall wound closure technique using barbed suture after SILA and compare perioperative outcomes with conventional method of layer by layer abdominal wall closure after SILA.

\section{MATERIALS AND METHODS}

We conducted a retrospective analysis for a total of 269 consecutive patients diagnosed with acute appendicitis who underwent SILS appendectomy from March 2014 to July 2016. Inclusion criteria were: patients who underwent single port laparoscopic appendectomy when acute appendicitis was suspected. The diagnosis of acute appendicitis was confirmed using contrast-enhanced abdominal CT or abdominal ultrasound. Single port laparoscopic appendectomy was chosen by patient preference after written informed consent was obtained in the case of acute appendicitis, including suppurative appendicitis and complicated type such as perforation.

Exclusion criteria were: (a) patients who had periappendiceal abscess and phlegmon, (b) those who underwent extensive surgery such as ileocecectomy and right hemicolectomy, (c) those who had appendiceal benign tumor, mucocele, or appendiceal cancer.

One hundred twenty-nine patients underwent continuous abdominal closure with V-Loc. They were compared with 140 patients who underwent conventional layer by layer abdominal wall closure technique. After obtaining approval from our Institutional Review Board, data about patients' demographics and perioperative outcomes including complications and cos$^{-}$ mesis were extracted and compared between the V-loc closure group and the conventional layer by layer closure group from medical records.

All operations were performed by a board-certified surgeon (Lim Sang Woo) in an emergency setting. Each patient was administrated routine intravenous $1.0 \mathrm{~g}$ of ceftriaxone from the time of diagnosis until postoperative 1 day. Diet such as sips of water, soft diet, and regular diet was started on postoperative one day depending on individual patient's general condition. Patients were discharged if they could tolerate ambulation and if regular diet was possible.

Preoperative demographics of patients included age, sex, body mass index $\left(\mathrm{kg} / \mathrm{m}^{2}\right)$, preoperative co-morbidity, American Society of Anesthesiologists (ASA) score, and history of previous abdominal surgery. Perioperative data during intraoperative period including abdominal wall closure time, insertion of an additional trocar, postoperative periumbilical incision length, pathologic report, time to resumption of diet, postoperative wound pain using a visual analogue scale (0: least pain; 10: worst pain), 30 day postoperative complications, and postoperative hospital stay were collected for both groups. The mean patient satisfaction score on a visual analogue scale survey related to periumbilical wound was assessed at one month after hospital discharge during an outpatient clinic visit (0: not satisfied; 10: fully satisfied).

\section{Surgical technique}

The patient was placed in supine position under general anesthesia. An approximately $1.5 \sim 2 \mathrm{~cm}$ infraumbilical transverse incision was made. The abdominal cavity was entered into using the open Hasson technique. Glove port (Nelis, Seoul, Korea) was then applied. After a pneumoperitoneum was created by insufflation of $8 \sim 12 \mathrm{mmHg} \mathrm{CO}_{2}$, a 30-degree 10-mm diameter rigid optic scope and conventional straight laparoscopic instruments were used for single incision laparoscopic appendectomy. Appendiceal localization and mobilization were performed in the routine manner. The mesoappendix including the appendiceal artery was divided using an ultrasonic dissector.

After double ligation of the appendiceal stump with endoloops, the appendix was transected and delivered extracorporeally into the vinyl pouch of the glove port. Copious saline irrigation and suction cleansing were performed at the surgical bed.

In cases of complicated appendicitis where postoperative abscess is a concern, a single closed suction Jackson-Pratt drain was occasionally inserted into the right lower quadrant of the abdomen.

Abdominal wall closure was performed according to the method for each group. The attending surgeon determined the time taken for abdominal wall closure from the fascia to the skin in both groups and the length of the periumbilical incision. The attending surgeon also took photographs of the incision. 


\section{The V-Loc closure group}

After removing the appendix, the divided peritoneum and the fascia of the linea alba were approximated and aligned. Closure was started at the end of the incision using unidirectional absorbable barbed suture V-Loc ${ }^{\mathrm{TM}} 90$ 2-0 (9" 23 $\mathrm{cm}$, Covidien, Mansfield, MA, USA) in a continuous running fashion. Closure technique used knotless, barbed 2-0 V-Loc 90 suture as shown by Demyttenaere et al. ${ }^{7}$

The first stitch with the needle for V-Loc passed through the loop of the thread. An additional stitch was applied over the fascia of the abdomen. A continuous running suture with tightening of each bite was performed at the opposite end of the incision. Again in the reverse direction, a continuous running suture was performed backward with reinforcement intent. After completing fascia closure, subcutaneous continuous running closure was performed using the same thread. For the skin layer, a subcuticular or an intradermal purse string suture along the incision line was performed with the remaining portion of V-Loc (Fig. 1). The thread containing needle was cut without tying a knot. Abdominal wall closure from the fascia to the skin was performed with a single thread of $\mathrm{V}$ Loc.

\section{The conventional layer by layer closure group}

After removing the inflamed appendix, three or four interrupted stitches using vicryl 2-0 sutures were performed through the external fascia. The subcutaneous fatty layer was closed with two or three stitches using fine 3-0 vicryl sutures. The skin was approximated and closed with a skin stapler (PROXIMATE ${ }^{\circledR}$ Fixed-head skin stapler, Ethicon, USA).

\section{Statistical analysis}

Categorical data are shown as percentages. Quantitative data are presented as mean values with standard deviations. Statistical analysis was performed using Student's t-test, Chi-square test, and Fisher's exact test. SPSS software version 17.0 (SPSS for Windows, Advanced Statistics, Inc., Chicago, IL, USA) was used for all statistical analyses. $p<0.05$ was considered statistically significant.

\section{RESULTS}

A total of 269 consecutive patients who underwent SILA from March 2014 to July 2016 were enrolled in the present study. Demographic data such as age, gender, and body mass index, history of previous abdominal operation, preoperative co-morbidity, or ASA score were not significantly different between the V-Loc closure group and the conventional layer by layer closure group (Table 1).

With respect to operation time, the V-Loc closure group showed shorter total operation time $(40.0 \pm 15.4 \mathrm{~min}$ vs. $44.9 \pm$ $16.3 \mathrm{~min}, p=0.013)$ and abdominal wall closure time $(5.5 \pm 0.9$ min vs. $6.5 \pm 0.8 \mathrm{~min}, p<0.001)$ than the conventional layer by layer closure group (Table 2). An additional trocar was inserted in $12(4.5 \%)$ cases among the entire cohort. No case of open
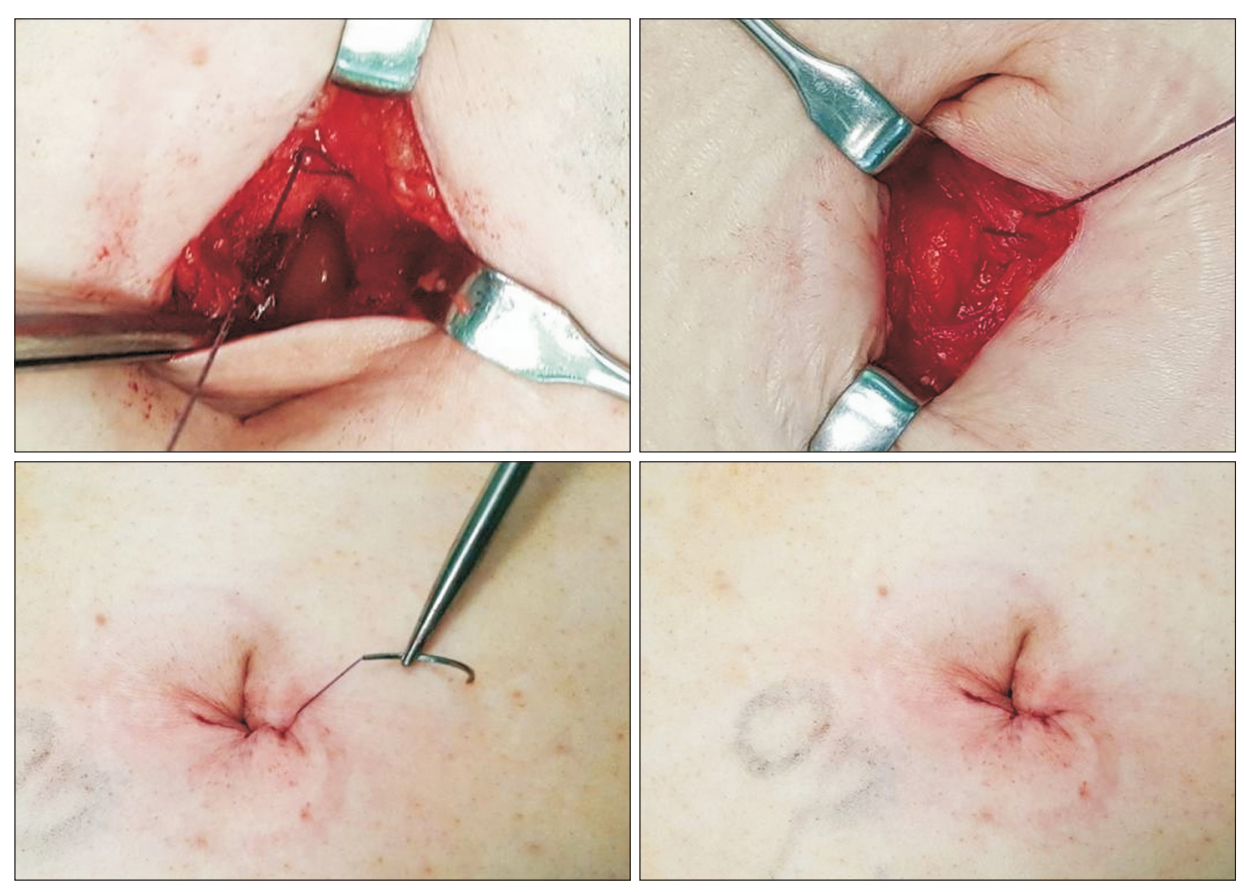

Fig. 1. Abdominal wall closure from the fascia to the skin was performed with a single $23 \mathrm{~cm}$ thread of V-Loc 90 including two layered overlapping fascia closure, subcutaneous fat approximation, and a continuous intradermal suture after single incision laparoscopic appendectomy. 
Table 1. Demographics of patients*

\begin{tabular}{|c|c|c|c|}
\hline & $\begin{array}{l}\text { V-LOC continuous closure } \\
\qquad(n=129)\end{array}$ & $\begin{array}{l}\text { Conventional layer by layer closure } \\
\qquad(n=140)\end{array}$ & $p$ value \\
\hline Mean age (years) & 41.3 (16.2) (range, $12 \sim 84$ ) & 39.2 (13.9) (range, $18 \sim 80$ ) & 0.252 \\
\hline Male sex & $73(56.6 \%)$ & $69(49.3 \%)$ & 0.231 \\
\hline Body mass index, $\mathrm{Kg} / \mathrm{m}^{2}$ & 23.7 (3.1) (range, 16.7 34.0) & 23.8 (3.4) (range, 16.3 35.1) & 0.848 \\
\hline Previous abdominal operation history & $6(4.7 \%)$ & $9(6.4 \%)$ & 0.526 \\
\hline Preoperative morbidity & $29(22.5 \%)$ & $23(16.4 \%)$ & 0.209 \\
\hline Hypertension & 18 & 16 & \\
\hline Diabetes mellitus & 12 & 9 & \\
\hline Old cerebrovascular disease & 3 & 2 & \\
\hline Asthma, COPD & 5 & 6 & \\
\hline Alcoholic hepatitis cirrhosis & 5 & 6 & \\
\hline Others & 6 & 4 & \\
\hline \multicolumn{4}{|l|}{ ASA score } \\
\hline 1/2/3 & $66 / 53 / 10$ & $79 / 57 / 4$ & 0.179 \\
\hline
\end{tabular}

${ }^{*}$ Values are expressed as mean and standard deviation or as number and percentage. BMI $=$ body mass index; ASA $=$ American Society of Anesthesiologists.

Table 2. Operative results*

\begin{tabular}{|c|c|c|c|}
\hline & $\begin{array}{l}\text { V-LOC continuous closure } \\
\qquad(\mathrm{n}=129)\end{array}$ & $\begin{array}{l}\text { Conventional layer by layer closure } \\
\qquad(\mathrm{n}=140)\end{array}$ & $p$ value \\
\hline \multicolumn{4}{|l|}{ Operative time (min) } \\
\hline Total operation time & 40.0 (15.4) (range, $20 \sim 100$ ) & 44.9 (16.3) (range, 25 85) & 0.013 \\
\hline Abdominal wall closure time & 5.5 (0.9) (range, $3 \mathrm{~min} 10 \mathrm{sec} \sim 9 \mathrm{~min} 21 \mathrm{sec}$ ) & 6.5 (0.8) (range, $4 \mathrm{~min} 20 \mathrm{sec} \sim 8 \mathrm{~min} 23 \mathrm{sec}$ ) & $<0.001$ \\
\hline Additional port insertion & $5(3.9 \%)$ & $7(5.0 \%)$ & 0.656 \\
\hline Drain insertion & $11(8.5 \%)$ & $13(9.3 \%)$ & 0.827 \\
\hline Postoperative incision length (cm) & $1.1(0.3)$ & $1.8(0.4)$ & $<0.001$ \\
\hline Pathologic finding & & & 0.132 \\
\hline Suppurative & $84(65.1 \%)$ & $103(73.6 \%)$ & \\
\hline Perforated & $45(34.9 \%)$ & $37(26.4 \%)$ & \\
\hline \multicolumn{4}{|l|}{ Postoperative pain $\left(V A S^{\#} 1 \sim 10\right)$} \\
\hline $12 \mathrm{hr}$ & $5.4(0.9)$ & $5.3(0.8)$ & 0.558 \\
\hline $24 \mathrm{hr}$ & $3.4(1.0)$ & $3.6(0.9)$ & 0.121 \\
\hline $48 \mathrm{hr}$ & $2.0(0.9)$ & $2.1(0.8)$ & 0.158 \\
\hline Time to resumption of diet (days) & $1.1(0.3)$ & $1.2(0.4)$ & 0.084 \\
\hline Hospital stay (days) & $3.6(2.5)$ & $4.0(1.5)$ & 0.096 \\
\hline
\end{tabular}

*Values are expressed as mean and standard deviation or as number and percentage. ${ }^{*}$ VAS = visual analog scale.

conversion was noted. Postoperative incision length assessed by the attending surgeon was significantly shorter in the VLoc closure group than that in the conventional layer by layer closure group (1.1 $\pm 0.3 \mathrm{~cm}$ vs. $1.8 \pm 0.4 \mathrm{~cm}, p<0.001)$.

The rate of perforated appendicitis in the pathologic report $(34.9 \%$ vs. $26.4 \%, p=0.132)$, postoperative wound pain at 
Table 3. Postoperative complications

\begin{tabular}{|cccc}
\hline & $\begin{array}{c}\text { V-LOC con- } \\
\text { tinuous closure } \\
(\mathbf{n}=129)\end{array}$ & $\begin{array}{c}\text { Conven- } \\
\text { tional layer by } \\
\text { layer closure } \\
(\mathbf{n}=140)\end{array}$ & $p$ value \\
\hline $\begin{array}{c}\text { Postoperative } \\
\text { complications }\end{array}$ & $8(7.0 \%)$ & $10(7.1 \%)$ & 0.758 \\
\hline $\begin{array}{c}\text { Periumbilical surgical } \\
\text { site infection }\end{array}$ & $4(3.1 \%)$ & $5(3.6 \%)$ & 0.830 \\
\hline $\begin{array}{c}\text { Incisional hernia } \\
\text { Appendiceal stump } \\
\text { leakage }\end{array}$ & 0 & $1(0.7 \%)$ & \\
\hline $\begin{array}{c}\text { Intra-abdominal pelvic } \\
\text { abscess }\end{array}$ & $2(1.6 \%)$ & $1(0.7 \%)$ & \\
\hline Postoperative ileus & 0 & $1(0.8 \%)$ & \\
\hline Urinary retention & $1(0.8 \%)$ & $1(0.7 \%)$ & \\
\hline
\end{tabular}

Values in parentheses are number $(\%)$.

postoperative 12 hours (5.4 vs. 5.3, $p=0.558$ ), 24 hours (3.4 vs. 3.6, $p=0.121$ ), or 48 hours ( 2.0 vs. $2.1, p=0.158$ ), time to resume diet (1.1 days vs. 1.2 days, $p=0.084$ ), or postoperative hospital stay (3.6 days vs. 4.0 days, $p=0.096$ ) was not significantly different between the V-Loc closure group and the conventional layer by layer closure group. There was no case of conversion to open surgery or mortality. Postoperative complications were comparable between the two groups $(7.0 \%$ in V-Loc closure group vs. $7.1 \%$ in conventional layer by layer closure group, $p=0.758$ ) (Table 3).

The rate of periumbilical surgical site infection was 3.1\% (4 cases) in the V-Loc closure group and 3.6\% (5 cases) in the conventional layer by layer closure group, showing no significant $(p=0.830)$ difference between the two groups. All periumbilical surgical site infections were of superficial incisional type.

One case of incisional hernia was found in the conventional layer by layer closure group. The patient was managed with incisional herniorrhaphy. Two cases of appendiceal stump leakage and one case of pelvic abscess were managed with conservative therapy including antibiotics.

Table 4 shows results of patient follow-up after discharge. There were three cases which needed outpatient clinic visits for wound dressing and two cases of readmission for radiologic interventional abscess drainage.

Mean patient satisfaction scores at 1 month after hospital discharge were collected during outpatient clinic visit or via telephone survey. A total of $175(65.1 \%)$ patients provided response. The mean patient satisfaction score was not sig-
Table 4. Patient follow-up after discharge

\begin{tabular}{|cccc}
\hline & $\begin{array}{c}\text { V.LOC } \\
\text { continuous } \\
\text { closure } \\
(\mathbf{n}=129)\end{array}$ & $\begin{array}{c}\text { Conventional } \\
\text { layer by } \\
\text { layer closure } \\
(\mathbf{n}=140)\end{array}$ & $p$ value \\
\hline $\begin{array}{c}\text { OPD re-visit for delayed } \\
\text { wound healing }\end{array}$ & $1(0.8 \%)$ & $2(1.4 \%)$ & 0.610 \\
\hline $\begin{array}{c}\text { Readmission for pigtail insertion } \\
\text { Patient satisfaction survey for } \\
\text { the wound after postoperative }\end{array}$ & $1(0.8(0.7)$ & $1(0.7 \%)$ & 0.954 \\
1 month (VAS 1 10) & & & \\
\hline
\end{tabular}

Values in parentheses are number (\%) or mean \pm standard deviation. "VAS = visual analogue scale.

nificantly different between the V-Loc closure group and the conventional layer by layer closure group $(8.8 \pm 0.7$ vs. $8.6 \pm 1.0$, $p=0.213$.

\section{DISCUSSION}

Single incision laparoscopic technique for appendicitis was introduced in the last decade. Since then, results have shown that SILA is a feasible, effective, and safe alternative of conventional three port laparoscopic appendectomy. In addition, it enables faster recovery. ${ }^{18}$

In the present study, abdominal wall closure after SILA was performed using commercially available barbed suture V-Loc, an absorbable wound closure device. In the published literature, wound closure with a barbed suture is comparable to the standard absorbable monofilament suture (Maxon) in terms of bursting strength and inflammatory response. However, it showed faster anastomosis time for gastrointestinal closure in animal models and human cadavers. ${ }^{7,19}$

Three commercially available barbed sutures have been currently introduced, Quill SRS bidirectional barbed suture (Angiotech, Vancouver, BC, Canada), Stratafix unidirectional or bidirectional barbed suture (Johnson \& Johnson, NJ, USA), and V-Loc unidirectional barbed suture (Covidien, Mansfield, MA, USA). Since 2013, V-Loc barbed suture has been used for laparoscopic surgeries including appendectomy, peptic ulcer perforation, abdominal wall closure after open surgery, esophagojejunostomy during gastric cancer surgery, and anastomosis reinforcement during colorectal cancer surgery at our institute.

V-Loc device consists of a helical barbed absorbable thread having a unidirectional barb with circumferential distribution having a loop at the end with a self-anchoring nature so that it does not loosen after initial tightening., ${ }^{8,11,15,20-22}$ These characteristics of $\mathrm{V}$-Loc device eliminate the need for tying a 
knot and reinforcement for preventing thread slippage. V-Loc maintains even tensile strength along the total length of the wound without knot tying. Thus, the time-consuming knot tying process at both ends of the suture line in the conventional layer by layer closure technique can be avoided.

In the present study, the time required for abdominal wall closure after specimen removal during SILA was shorter in the V-Loc closure group than that in the conventional layer by layer closure group. During the intradermal suturing procedure for skin closure, tightening the thread using its characteristic of self-anchoring nature reduced the postoperative incision length compared to the conventional layer by layer closure.

In the present study, postoperative recovery and wound pain were similar in both V-Loc and conventional layer by layer closure groups. Postoperative complications including periumbilical surgical site infection were also comparable between the two groups. Gentle and minimal manipulation of the thread during wound closure following the surgical principle may minimize surgical site infection in the situation of emergency appendectomy. Trocar site incisional hernia may cause intestinal evisceration or mechanical obstruction. A case of postoperative incisional hernia was not found in the $\mathrm{V}$-Loc closure group in the present study. Patients enrolled in the present study were non-obese East Asians with a median BMI of $23 \sim 25 \mathrm{~kg} / \mathrm{m}^{2}$. This selection bias may be one of the factors responsible for few events of incisional hernia. Further randomized controlled study is warranted. Modified suturing with a reinforcement intent might be applicable for abdominal wall closure.

In the present study, we applied a barbed suture for abdominal wall closure after single port laparoscopic surgery. The time required for abdominal wall closure was shorter and the technique was easier compared to the conventional layer by layer abdominal wall closure technique. Although we adopted the V-Loc for small wound after SILA, our operative findings and results agree with previous findings reported for dermal closure of Pfannenstiel incisions ${ }^{20}$ and body-contouring. ${ }^{23}$ The finding of shrinkage or smaller wound in the present study might offer potential advantage of cosmesis, although the result of patient satisfaction survey was similar to that after conventional layer by layer closure. Indications for use of a barbed suture might be extended to abdominal fascia wall closure based on our findings.

From the perspective of laparoscopic suturing which is a very difficult procedure with high technical demands, many published articles have reported significant reduction in suture time in the barbed group during laparoscopic intracorporeal procedures for gastrointestinal tract surgery and gynecology surgery. ${ }^{11,12,21,24,25}$ The non-requirement of tying a knot after every continuous or interrupted suture for maintaining tight tension is a major advantage of using a barbed suture. It can shorten the operation time during both laparoscopic gastrointestinal and gynecological suturing.

The cost of a barbed suture has been reported to be high for laparoscopic intracorporeal suturing. ${ }^{26}$ In the present study, the cost for single use of a barbed suture in South Korea was not greater than that of using two or three threads of vicryl in the conventional layer by layer closure technique ( $\$ 12.99$ vs. \$19.92). Therefore, V-loc barbed suture might be applicable for abdominal closure after SILA which is classified as a diagnosis-related group (DRG) under the coverage of the national health insurance system in South Korea.

The reported disadvantage of a barbed suture is bowel obstruction caused by grasping the surrounding tissue. ${ }^{27}$ When using a barbed suture in intracorporeal intestinal closure, cautious consideration is warranted. Cutting the remnant suture end short may prevent adhesion-related intestinal obstruction. However, unique characteristics of a barbed suture of grasping the surrounding tissue, increasing tissue surface adhesion, and distribution of tension might be advantageous factors for abdominal wall closure as found in the present study.

Single use of $23 \mathrm{~cm}$ long commercial V-Loc 90 is enough to allow for a multi-layered suture including two layered overlapping fascia closure, subcutaneous fat approximation, and a continuous intradermal suture with remnant portions of the remaining threads in a SILA situation while reducing incision length as shown in the present study.

In the present retrospective cohort study, we aimed to evaluate the safety and feasibility of knotless unidirectional barbed sutures for closure of the abdominal wall after a minimally invasive surgery such as laparoscopic appendectomy. In a consecutive series of a single institute experience, barbed sutures of V-Loc needed less time for single site laparoscopic wound closure with reduced costs and comparable morbidities of surgical site infection and potential incisional hernia compared to conventional layer by layer closure.

Based on the results of the present study, knotless barbed sutures with the characteristics of resistance to slippage for abdominal wall closure can be applied in difficult trocar-site closure after laparoscopic surgery in morbidly obese patients during situations that is difficult to tie a knot in a limited space with visual field. Moreover, it may be a preferred substitute for reinforcement after fascial layer closure over a retention suture even after emergency exploratory laparotomy.

Limitations of the present study include the non-randomized, retrospective nature, small sample size, and a single surgeon's experience. The enrolled South Korean patients were basically slender, non-obese East Asians. Thus, selection bias might have occurred. The shorter postoperative incision length might affect postoperative pain score and subjective 
satisfaction survey as a confounder.

We compared barbed suture method with conventional interrupted suture technique. A comparison with continuous running suture techniques may be possible in future reports. We are planning to make such comparison. Outcomes of long-term follow-up data for a barbed suture are warranted. An assessment of objective measurement for technical ease or difficulty is also necessary. A larger scale, prospective randomized controlled trial is warranted.

In conclusion, a unidirectional knotless barbed suture is an effective method for abdominal wall closure after periumbilical single incision laparoscopic surgery. The barbed suture technique is safe, convenient, time saving, and well tolerated after trocar site closure. Also, short-term operative outcomes including surgical site infection and incisional hernia of this method are comparable to those of conventional layer by layer closure method. Therefore, babed suture technique might be a useful alternative of conventional layer by layer closure because it can provide comparable cosmesis.

\section{REFERENCES}

1) Kouhia ST, Heiskanen JT, Huttunen R, Ahtola HI, Kiviniemi VV, Hakala T. Long-term follow-up of a randomized clinical trial of open versus laparoscopic appendicectomy. Br J Surg 2010;97:1395-1400.

2) Kang BM, Hwang JW, Ryu BY. Single-port laparoscopic surgery in acute appendicitis: retrospective comparative analysis for 618 patients. Surg Endosc 2016;30:4968-4975.

3) Kotaluoto S, Pauniaho SL, Helminen M, Kuokkanen H, Rantanen T. Wound healing after open appendectomies in adult patients: a prospective, randomised trial comparing two methods of wound closure. World J Surg 2012;36:2305-2310.

4) Andrade LA, Munoz FY, Baez MV, et al. Appendectomy Skin Closure Technique, Randomized Controlled Trial: Changing Paradigms (ASC). World J Surg 2016;40:2603-2610.

5) Koskela A, Kotaluoto S, Kaartinen I, Pauniaho SL, Rantanen T, Kuokkanen H. Continuous absorbable intradermal sutures yield better cosmetic results than nonabsorbable interrupted sutures in open appendectomy wounds: a prospective, randomized trial. World J Surg 2014;38:1044-1050.

6) Chiang RA, Chen SL, Tsai YC. Delayed primary closure versus primary closure for wound management in perforated appendicitis: a prospective randomized controlled trial. J Chin Med Assoc 2012:75:156-159.

7) Demyttenaere SV, Nau P, Henn M, et al. Barbed suture for gastrointestinal closure: a randomized control trial. Surg Innov 2009; 16:237-242.

8) Lee SW, Kawai M, Tashiro K, et al. Laparoscopic gastrointestinal anastomoses using knotless barbed absorbable sutures are safe and reproducible: a single-center experience with 242 patients. Jpn J Clin Oncol 2016;46:329-335.

9) Yang SM, Hsiao WL, Lin JH, Huang PM, Lee JM. Laparoscopic percutaneous jejunostomy with intracorporeal V-Loc jejunopexy in esophageal cancer. Surg Endosc 2017;31:2678-2686.

10) Son SY, Cui LH, Shin HJ, et al. Modified overlap method using knotless barbed sutures (MOBS) for intracorporeal esophagojejunostomy after totally laparoscopic gastrectomy. Surg Endosc 2017;31:2697-2704.

11) Cong L, Li C, Wei B, Zhan L, Wang W, Xu Y. V-Loc 180 suture in total laparoscopic hysterectomy: a retrospective study comparing Polysorb to barbed suture used for vaginal cuff closure. Eur J Obstet Gynecol Reprod Biol 2016;207:18-22.

12) Song T, Lee SH. Barbed suture vs traditional suture in singleport total laparoscopic hysterectomy. J Minim Invasive Gynecol 2014;21:825-829.

13) Kim JH, Byun SW, Song JY, et al. Barbed versus conventional 2-layer continuous running sutures for laparoscopic vaginal cuff closure. Medicine (Baltimore) 2016;95:e4981.

14) Kim EY, Hong TH. Laparoscopic Longitudinal Pancreaticojejunostomy Using Barbed Sutures: an Efficient and Secure Solution for Pancreatic Duct Obstructions in Patients with Chronic Pancreatitis. J Gastrointest Surg 2016;20:861-866.

15) Fernandez LC, Toriz A, Hernandez J, et al. Knotless choledochorraphy with barbed suture, safe and feasible. Surg Endosc 2016;30:3630-3635.

16) Lee JS, Yoon YC. Laparoscopic common bile duct exploration using V-Loc suture with insertion of endobiliary stent. Surg Endosc 2016;30:2530-2534.

17) Rubin JP, Hunstad JP, Polynice A, et al. A multicenter randomized controlled trial comparing absorbable barbed sutures versus conventional absorbable sutures for dermal closure in open surgical procedures. Aesthet Surg J 2014;34:272-283.

18) Xu AM, Huang L, Li TJ. Single-incision versus three-port laparoscopic appendectomy for acute appendicitis: systematic review and meta-analysis of randomized controlled trials. Surg Endosc 2015;29:822-843.

19) Nemecek E, Negrin L, Beran C, Nemecek R, Hollinsky C. The application of the V-Loc closure device for gastrointestinal sutures: a preliminary study. Surg Endosc 2013;27:3830-3834.

20) Murtha AP, Kaplan AL, Paglia MJ, Mills BB, Feldstein ML, Ruff GL. Evaluation of a novel technique for wound closure using a barbed suture. Plast Reconstr Surg 2006;117:1769-1780.

21) Fouda UM, Elsetohy KA, Elshaer HS. Barbed Versus Conventional Suture: A Randomized Trial for Suturing the Endometrioma Bed After Laparoscopic Excision of Ovarian Endometrioma. J Minim Invasive Gynecol 2016;23:962-968.

22) Watanabe G, Ishikawa N. Use of barbed suture in robot-assisted mitral valvuloplasty. Ann Thorac Surg 2015;99:343-345.

23) Duscher D, Pollhammer MS, Wenny R, Shamiyeh A, Schmidt 
M, Huemer GM. Barbed Sutures in Body-Contouring: Outcome Analysis of 695 Procedures in 623 Patients and Technical Advances. Aesthetic Plast Surg 2016;40:815-821.

24) Song T, Kim TJ, Kim WY, Lee SH. Comparison of barbed suture versus traditional suture in laparoendoscopic single-site myomectomy. Eur J Obstet Gynecol Reprod Biol 2015;185:99-102.

25) Bulbuller N, Aslaner A, Oner OZ, et al. Comparison of four different methods in staple line reinforcement during laparascopic sleeve gastrectomy. Int J Clin Exp Med 2013;6:985-990.
26) Alessandri F, Remorgida V, Venturini PL, Ferrero S. Unidirectional barbed suture versus continuous suture with intracorporeal knots in laparoscopic myomectomy: a randomized study. J Minim Invasive Gynecol 2010;17:725-729.

27) Oor J, de Castro S, van Wagensveld B. V-loc capable of grasping surrounding tissue causes obstruction at the jejunojejunostomy after Roux-en-Y laparoscopic gastric bypass. Asian J Endosc Surg 2015;8:209-211. 